\title{
Filigrane
}

Écoutes psychothérapiques

\section{Entre honte et psychose : réflexions sur un paradoxe social}

\section{Delphine Scotto Di Vettimo}

Volume 15, numéro 2, automne 2006

URI : https://id.erudit.org/iderudit/014467ar

DOI : https://doi.org/10.7202/014467ar

Aller au sommaire du numéro

Éditeur(s)

Revue Santé mentale au Québec

ISSN

1192-1412 (imprimé)

1911-4656 (numérique)

Découvrir la revue

Citer cet article

Scotto Di Vettimo, D. (2006). Entre honte et psychose : réflexions sur un paradoxe social. Filigrane, 15(2), 81-93. https://doi.org/10.7202/014467ar

\section{Résumé de l'article}

L'auteur se réfère d'abord à son étude sur le statut métapsychologique de la honte ; il en situe les enjeux et les limites et propose ici une discussion critique de la problématique de la honte considérée sur le versant social où elle fait symptôme. L'hypothèse clinique qui guide son propos postule que dans le travail auprès de sujets confrontés à une expérience psychotique, l'expression de la honte comme épreuve ontologique au regard de l'Autre constituerait une tentative du sujet de s'éprouver comme tel. Cette réflexion insiste, à travers une évocation clinique, sur cette co-occurrence entre honte, psychose et lien social. 


\title{
Entre honte et psychose: réflexions sur un paradoxe social
}

\author{
delphine scotto di vettimo
}

\begin{abstract}
L'auteur se réfère d'abord à son étude sur le statut métapsychologique de la honte; il en situe les enjeux et les limites et propose ici une discussion critique de la problématique de la honte considérée sur le versant social où elle fait symptôme. L'hypothèse clinique qui guide son propos postule que dans le travail auprès de sujets confrontés à une expérience psychotique, l'expression de la honte comme épreuve ontologique au regard de l'Autre constituerait une tentative du sujet de s'éprouver comme tel. Cette réflexion insiste, à travers une évocation clinique, sur cette co-occurrence entre honte, psychose et lien social.
\end{abstract}

«Par la honte, le sujet témoigne [...] qu'il demeure sujet. Il se reconstruit, autour de la blessure d'idéal, en témoignant Qu'il reste en souffrance d'idéal» (Assoun, 1999, 109).

\section{Introduction}

e Dictionnaire de la langue française d'Émile Littré propose la définition suivante de la honte: «Déshonneur, honte, humiliation» et reprend aussi, en termes quasi similaires, la définition du Dictionnaire de l'Académie française qui s'énonce ainsi : "Confusion, trouble, sentiment pénible excité dans l'âme par l'idée de quelque déshonneur qu'on a reçu ou qu'on craint de recevoir, ou qu'on aurait seulement à ses propres yeux». Il convient de noter que la distinction entre le psychique et le social n'est pas clairement établie, distinction qui est capitale dans l'étude de la honte. L'usage de termes tels que «confusion», «sentiment» et «crainte» souligne le caractère psychique de la honte par rapport à son caractère social. Il conviendra de revenir sur ce statut assez paradoxal de la honte. Retenons, pour l'instant, que la honte est d'abord un sentiment social: elle apparaît le plus souvent en réaction au regard d'autrui et vient marquer «l'échec de la confirmation narcissique» (Miollan, 1998, 163). Cette primauté du regard dans l'expérience de honte, trouverait son hypothèse interprétative dans le fait que la honte prendrait sa source dans le regard de l'Autre, regard qui révélerait au sujet ses propres limites, son incomplétude, autrement dit dévoilerait le décalage insoutenable entre l'image narcissique de soi faite de perfection et une image reconnue dans le regard de l'Autre, d'insuffisance et d'imperfection. Nous nous situons là dans la perspective lacanienne classique qui postule, au sujet de l'Autre, que « [...] c'est de lui que le sujet se constitue, par quoi c'est de l'Autre que le sujet reçoit 
même le message qu'il émet» (Lacan, 1960, 807). La décharge fulgurante de honte telle qu'elle a été décrite chez Sartre dans une perspective phénoménologique, témoigne fort justement de cette expérience émotionnelle où une part du plus intime de soi se trouve brutalement exposée, véritable mise à nu du sujet qui le confine dans un chaos narcissique. Dans l'expérience de honte, l'effet de transparence ressenti en est la traduction la plus explicite, qui ne saurait mieux s'exprimer que dans l'expression langagière, assez coutumière, de mise à nu. Ce ravalement à une fonction d'objet engendre un grand désarroi chez le sujet, car il est alors en proie à une incertitude narcissique angoissante. En ce sens, la honte constitue une expérience subjective singulière, celle d'un sujet brusquement dévoilé dans son intimité de pensée, par la présence d'un regard dont il est impossible de se dérober. En tant qu'affect, la honte est d'emblée sociale, étroitement liée au regard de l'autre, tout autant qu'expression de la manifestation d'une revendication singulière. Nous rejoignons ici l'analyse de S. Tisseron qui rend compte d'une variété d'explications de la honte mais surtout de sa dimension sociale, dans le sens où elle n'est pas seulement un sentiment subjectif mais aussi un sentiment «transsubjectif» qui fait lien (Tisseron, 1992, 178). Nous aurons l'occasion d'y revenir.

Ce travail est une réflexion élaborée, d'une part à partir de la question du statut métapsychologique de la honte dans l'œuvre freudienne, de ses manifestations et de son traitement psychothérapique (Scotto Di Vettimo, 2001) et d'autre part à partir de la question de l'appréhension de la honte dans le champ de la psychose. Il s'agit ici, à partir d'une situation clinique, de questionner l'épreuve de honte chez un sujet psychotique et plus largement d'interroger la problématique de la honte considérée sur le versant social où elle fait symptôme. Pour le dire en d'autres termes, il s'agit de venir éclairer ce qu'il en est des conditions de maintien de la subjectivité quand elle est en menace d'anéantissement du fait même du processus de désymbolisation psychotique, destructeur des repères identificatoires tels qu'ils fondaient la place du sujet dans l'ordre de l'humain. Dans cette perspective, et ce sera ici le propos, il convient de questionner la honte comme forme d'expression narcissique: le sujet trouve un accès à la subjectivation dans et par l'épreuve de honte.

\section{Position du problème}

La référence à la honte n'est pas rare dans l'œuvre de Freud et s'appréhende globalement comme indice d'un fonctionnement sur le mode narcissique. Dans L'interprétation des rêves (Freud, 1900, 213) Freud souligne le caractère spéculaire de la honte, qui tient au regard: la honte se nourrit de l'incomplétude imaginaire qui constitue l'objet même de l'intérêt et de l'amour narcissique, en référence à une représentation de soi et de l'épreuve que représente le fait de se reconnaître imparfait, limité, manquant, c'est-à-dire soumis à l'épreuve de la castration.

Mais, et de façon plus large, le mot allemand Scham désigne dans l'usage freudien, aussi bien la honte comme formation réactionnelle dans la névrose 
obsessionnelle, comme «digue psychique ( Freud, 1905, 101) avec le dégoût et l'exigence d'idéal esthétique et moral, et dont la mission est de faire rempart à l'envahissement pulsionnel, notamment des pulsions sexuelles de voyeurisme et d'exhibitionnisme au début de la période de latence. Or, et c'est là un point essentiel, ce développement souligne d'une part le caractère de proximité de l'affect de honte avec le champ du pulsionnel qu'il est chargé de contenir, et d'autre part son aspect brut, non élaboré et non maîtrisable. Bien avant de revêtir ce caractère de formation réactionnelle et de «digue psychique» dans le développement de l'enfant, Freud situe l'émergence de honte dans les moments où la satisfaction pulsionnelle de l'enfant tombe sous le regard de l'autre, cet autre qui, dans l'instant même de l'énoncé de l'interdit et avant même que cet énoncé ne fasse sens, devient un adulte honnisseur. Ici, la honte est en rapport avec un objet honni (l'exhibition), objet d'opprobre car il touche au plaisir scopique, au corps, à l'intimité et au sexuel. Cette honte très tôt ressentie — préœdipienne - témoigne alors de l'importance du regard tiers, de sa fonction potentiellement castratrice, et de l'intransigeance parentale face à la quête infantile de satisfaction pulsionnelle. En écho, la honte témoignerait alors que dans l'après-coup de cet énoncé vécu sur un mode purement arbitraire, pur effet de coupure, le regard de l'autre prendrait une dimension de toute-puissance voire même persécutrice; à ce moment précis, aucune élaboration symbolique ne viendrait en atténuer les effets. Toute la question posée par la honte réfère bien à son caractère fondamentalement intersubjectif, à savoir qu'elle accompagne un échec devant témoin et conduit à vouloir le cacher.

Au-delà, dans l'œuvre de Lacan, la honte est considérée comme ontologique puisqu'elle est articulée à l'Homme. Cette hypothèse paraît s'accorder avec l'hypothèse freudienne sur la honte. À l'appui de ces considérations, l'émergence de honte ne porterait donc pas seulement sur le sentiment de valeur et d'estime de soi mais aussi et surtout sur le sentiment d'identité, dont la honte en révèlerait les fissures et les ruptures. C'est précisément dans cette occurrence qu'il s'agit de penser l'existence de honte chez le psychotique. Cette conviction constitue une ligne d'écoute du discours de sujets confrontés à une expérience psychotique et hospitalisés en psychiatrie. Quand une parole peut être énoncée à sa suite, on peut constater qu'elle se déploie dans le transfert pour dire une sorte d'indigence. Parler de sa problématique psychotique est alors avant tout se dire différent, décalé, dévalorisé et irrémédiablement abîmé. Par ailleurs, des expressions telles que «étrangeté», «errance», «exclusion» émaillent les entretiens avec de tels patients; autant d'expressions qui témoigneraient de ce recouvrement de la réalité par un réel qui submerge l'appareil psychique et ses constructions imaginaires et qui signent, à plus ou moins brève échéance, le glissement de la potentialité psychotique à la décompensation psychotique (Aulagnier, 1984, 16). Cette involution inhérente à l'entrée dans la psychose perdrait le sens de ce qui, dans l'éprouvé de honte, fonctionnerait comme trace, soutenant ainsi l'essentiel de son enjeu subjectif, soit sa survenue comme sauvegarde narcissique. 
Dans son ouvrage intitulé Le Préjudice et l'Idéal, P.-L. Assoun propose de considérer — « [...] des exilés au sens propre — délocalisation géographique et sociale - et des exilés au sens figuré - désubjectivation et malaise de l'intériorité » (Assoun, 1999, 114). C'est précisément ce dernier argument qui nous intéressera ici au titre de ce qu'il révèle de l'expérience psychotique comme épreuve de désubjectivation et d'exil intérieur. Comment un sujet peut-il être confronté, de l'intérieur, à ce sentiment d'être ailleurs, étranger, exilé? Une clinique de l'exil dans le champ de la psychose pourrait interroger cette épreuve d'étrangeté qui tient à une position subjective. Parler «d'exil intérieur»(Assoun, 1999, 114) permettrait de désigner une sorte de métaphore, celle qui assigne le psychotique à cette expérience — le plus souvent terrifiante — de dépossession de soi, d'exclusion et de désubjectivation.

J'ai été sensibilisée - comme d'autres praticiens - à l'expression de sentiments de honte qui pouvaient colorer le récit d'expériences de sujets psychotiques et qui surtout semblaient envahir, invalider, l'ensemble de leur vie psychique, de leur expérience relationnelle et sociale. Non seulement la honte gagnait, envahissait la sphère subjective, mais la persistance devenait répétition dans la mise en scène de récits de situations productrices de honte pour ces sujets. Pourquoi l'expérience psychotique produit-elle de la honte chez le sujet? L'hypothèse ici envisagée postule que dans le travail clinique, l'expression de la honte comme épreuve ontologique au regard de l'Autre constituerait une tentative du sujet de s'éprouver comme tel et de maintenir un lien social.

En effet, si, pour le sujet, l'expression de la honte est porteuse d'une acception précise, elle est pour le clinicien d'abord indice et messagère d'une signification particulière donnée à la parole (Scotto Di Vettimo, Jacobi, 2003, 112). Cette interrogation va trouver certains prolongements du point de vue de son usage dans le discours, par exemple, un sujet qui dit qu'il a un sentiment de honte, n'est pas nécessairement affecté par la honte. Autrement dit, l'usage du terme de honte par le sujet ne préjuge pas de l'existence de l'affect. Comme l'écrit B. Jacobi, «Si elle est exposition, exhibition, la parole se différencie radicalement de ce qu'elle évoque » (Jacobi, 2000, 151). Dès lors, la question qui, pour le clinicien, se pose, est bien justement, de discerner l'épreuve de honte d'un sujet au-delà des termes du discours qui accompagne cette épreuve. Il est vrai que l'usage de ce terme ne renvoie pas à un diagnostic, à une nosographie. Mais il mérite à s'expliquer sur le saut qui consiste à affirmer la présence de honte pour un sujet à partir de l'occurrence de ce terme dans son discours.

Je propose, dans cet article, de situer des développements et des connexions de cette prise de position à partir d'une situation clinique de rupture plus ou moins radicale : le discours psychopathologique d'une jeune femme qui traduit son entrée dans la psychose. À dessein, un bref extrait du travail thérapeutique permettra d'une part de revenir sur l'hypothèse de travail indiquée au début de cet article et d'autre part de porter une attention particulière sur certaines modalités du processus psychotique et de l'expérience de honte qui lui est contingente. 
Une jeune femme que nous appellerons Magali a été accueillie dans un atelier thérapeutique d'insertion socioprofessionnelle, à la suite d'une hospitalisation en psychiatrie d'une durée de douze mois pour «décompensation psychotique».

\section{Magali ou la permanence d'un sentiment de honte Données biographiques}

Magali, âgée de 32 ans, est la cadette d'une fratrie de deux enfants: elle a un frère aîné qui est cadre supérieur dans une entreprise. Elle passe régulièrement les week-ends chez ses parents qui sont tous deux retraités.

Les éléments historiques et cliniques recueillis font mention d'une «mauvaise entente familiale » et témoignent — concernant Magali — «d'une enfance plutôt difficile, une bonne élève mais toujours en retrait et décrite comme apathique».

Les premiers troubles psychopathologiques apparaissent alors que Magali a 18 ans et qu'elle est inscrite en première année de Faculté de Droit. Elle présente à cette période un désintérêt intellectuel, des idées délirantes de transformation corporelle, un isolement affectif et social ainsi que des manifestations de violence envers sa famille. Après plusieurs mois d'hospitalisation dans un service de psychiatrie, le diagnostic médical retenu est le suivant: psychose schizophrénique.

\section{Rencontres cliniques}

À son arrivée à l'atelier thérapeutique, Magali sollicite des entretiens en raison de questionnements obsédants sur l'image de soi - en particulier son corps et sa peau - le tout sur fond d'inhibitions phobiques qui envahissent son champ d'activités: elle craint d'aller au devant des gens, en l'occurrence ici les autres patients, car elle redoute que ces derniers ne se moquent d'elle. Je suis d'emblée frappée par son allure: elle a les cheveux coupés très courts et porte une large salopette en jean assortie de baskets. Son corps semble littéralement disparaître dans ces vêtements bien trop amples. Lors du premier entretien, Magali parle de façon monocorde et triste, résumant sa situation actuelle dans une sorte de récapitulation sommaire évoquée en ces termes : «Ma maladie est grave : je prends du poids et j'ai une tendance à me laisser aller. » L'entretien sera ponctué de longs silences durant lesquels Magali imprime à son corps un balancement d'avant en arrière, tout en maintenant rivés ses yeux sur le parterre.

Dans les rencontres qui suivent, Magali parle assez facilement d'elle : comment elle était «avant» et comment, en peu de temps, sa vie a changé. Elle ne saurait dire comment tout cela lui est venu. Peu à peu et, en même temps, très subitement; elle avait le souvenir d'avoir été une enfant «comme les autres» mais depuis ces multiples hospitalisations, elle dit avoir beaucoup perdu. L'origine de sa maladie, selon elle, est à relier sans conteste à l'absence d'amour parental; elle confie : «Mes parents ne sont pas attachés à moi, cela m'handicape et me rend malade, de boulimie surtout. Pire, cela a entraîné mon désœeuvrement et ma déperdition dans les rues de la ville». Dans ses souvenirs, les premiers symptômes seraient apparus alors qu'elle était à l'université : «La réalité et les échanges sociaux — dit-elle — 
sont devenus de plus en plus difficiles: une frontière est imposée, une limite. Ce que je vivais catastrophiquement, c'est ma solitude et mes rapports avec les autres. Les échanges humains devenaient de plus en plus pauvres. » Chacun de ses récits est empreint de malaises récurrents, rapporte un climat de désarroi et d'impuissance. Elle décrit alors une perte progressive de confiance en elle-même, un repli et un isolement social de plus en plus importants : «Je me sentais dans une situation minable à cause de ma santé défaillante, de l'inégalité sociale et de cette solitude qui me pesait». C'est alors qu'elle relate le début de son errance dans les rues de la ville: «Par réaction, je partais dans la ville, j'errais, tout cela a provoqué un détraquement. » La décompensation suivra deux mois plus tard. Elle sera alors Hospitalisée à la Demande d'un Tiers (H.D.T.), son père en l'occurrence, dans un service psychiatrique.

Aujourd'hui, la psychose est bien là dans toute sa masse physique et psychique. Elle voudrait bien ne plus penser à sa maladie et à ses inévitables conséquences qu'elle évoque ainsi : «Je me sens déracinée ». En certaines occasions, elle fait part de questionnements existentiels qui témoignent de sa tentative pour donner un sens à sa vie.

Dans les mois qui suivent et alors que les entretiens se poursuivent à leur rythme habituel, elle pourra aborder plus précisément un vécu de honte qu'elle associe aux effets de perte, subjective et narcissique, que sa maladie a provoqués. Voici ce qu'elle dit: «J'ai honte en permanence de ma maladresse, je suis malhabile et ignorante. Ma maladie a provoqué une dégradation intime. J'ai honte de moi ». Ici, nous pouvons entendre cette dénomination des affects comme découverte que les mots entraînent avec eux, dès lors que la parole leur restitue la capacité de nommer.

C'est ainsi que toute une déclinaison de hontes dans des registres très variés va se déployer au fil des entretiens. Le sentiment de honte semble réunir ces expériences de l'enfance et de l'adolescence. Honte du désamour parental, honte de se sentir différente des autres, honte d'avoir été malmenée, honte de son échec universitaire. La description par Magali de ce vécu symptomatique occupera, durant de nombreux mois, le devant de la scène. Tout se passe comme si cette description était faite pour se reconnaître dans ce qu'elle est devenue et tenter de s'approprier cette nouvelle image d'elle-même. Nous pourrions entendre en ce sens que le huis clos de l'état psychotique n'est pas seulement dans le sentiment d'isolement; il est fait aussi de cette dépossession de l'image de soi : aucun miroir ne peut renvoyer sa propre image et, à la limite, s'y reconnaîtrait-elle ? En outre, le sentiment de dévalorisation de soi renforce cette idée qu'il n'est plus possible d'entreprendre et de penser les choses comme avant. À propos de cette contiguïté clivée, elle confiera que sa pensée interroge en permanence — sous forme de ruminations obsédantes — «l'avant» et «l'après» maladie, la psychose ayant porté atteinte, selon elle, à son imagination, sa capacité de rêverie, sa capacité de penser et d'agir. Nous pouvons aller au-delà et rappeler que l'économie psychotique est aussi corrélative d'une atteinte à la représentation et l'action, aux 
potentialités du langage, ainsi qu'à la communication intersubjective. Mais plus. À l'identité subjective. De cette restriction et de cet appauvrissement de sa sphère psychique, elle confiera ressentir la preuve de son aspect irréversible: c'est le sentiment que le temps s'est complètement modifié, où présent, passé, futur se télescopent et où elle ne sait alors plus «qui elle est». Cet écrasement des temps intérieurs de la vie psychique serait à entendre comme collusion et déchirure du tissu temporel, où réalité historique et réalité actuelle viennent à se confondre comme l'envers et l'endroit d'un même processus.

Mais comment désigner l'unité d'une structure psychotique dans sa perturbation? Magali exprime la nostalgie de sa «normalité», lorsqu'elle se vivait dans une capacité d'agir, d'anticiper se renouvelant d'elles-mêmes en agissant et dans une mobilité physique et psychique qui lui donnait le plaisir, entre autres, d'initiatives et d'activités de loisirs diverses.

Le plus souvent, lorsqu'elle revient sur sa maladie, elle interprète son discours intérieur comme une sorte de commentaire sans fin, comportant un regard désapprobateur sur ce qu'elle est devenue. Elle ajoutera par la suite que cette façon intérieure de ressasser l'amène à ressentir qu'elle se fige de plus en plus dans un immobilisme qui n'a plus rien d'humain; elle confie: «Je me sens diminuée par rapport à la maladie... en fait, je suis réellement diminuée ». Le constat de ces insuffisances débouche sur la conscience accrue et cruelle d'une défaillance irrémissible.

Mais plus fondamentalement, la question qui se pose, pour Magali, au regard de sa maladie et de ses effets de désubjectivation, est bien celle de ses radicales conséquences: changement des capacités physiques et intellectuelles, quand elle réalise qu'elle ne peut plus faire ce qu'elle faisait avant (deuil des études notamment), tout ce qui la liait aux autres de sa génération (perte des camarades de l'université), tout ce que les parents attendaient d'elle (ils souhaitaient qu'elle fasse des études universitaires), aimaient en elle.

\section{D'une clinique de la honte à l'(h)ontologie}

L'analyse de ce fragment clinique permet à présent de revenir sur l'affirmation indiquée au début de cet article, à savoir que l'une des fonctions de la honte, c'est de s'éprouver comme sujet. Reprendre les mots de l'épreuve psychotique qui vise le tréfonds de l'être, ces mêmes mots qui témoignent de cette souffrance vive et aliénante — «Le problème — confiait Magali — c'est cet isolement de ma personne, le fait de vouloir vivre en groupe et d'être rejetée, exclue par le groupe »nous amène à l'exploration du sentiment d'étrangeté et d'exclusion propres à la psychose. C'est sur ce fond de souffrance inhérent au processus psychotique, sur l'impossibilité de s'en rendre maître que P.-L. Assoun évoque la «sensation paradoxale d'être inclus dans un espace qui, hostile ou «accueillant» en lui-même, ne permet pas à son autre de s'y reconnaître, donc l'exclut» (Assoun, 1999, 114). Ce dont Magali témoignait en ces termes : «Les autres sont différents de moi, ils ont quelque chose de plus. » 
Le sentiment d'exclusion viendrait attester d'un moment où vacille la certitude d'un lien social: «Point d'exclusion sociale sans un sujet qui produit le geste de s'exclure » (Assoun, 1999, 36). C'est précisément ce que relatait Magali pour justifier le maintien délibéré de son isolement; par exemple par rapport aux autres patients de l'atelier qui organisaient des soirées et des sorties auxquelles elle était conviée mais qu'elle déclinait systématiquement. Cette conviction d'un préjudice social comme «handicap» pour reprendre ses propres termes, se redoublait d'un autre préjudice à vocation délirante — qu'elle exprimait de la manière suivante: «Je cherche une vérité sur ma vie personnelle qu'on ne veut pas m'accorder, une vérité sur mon identité, ma vie biologique... les gens savent quelque chose de moi que j'ignore. C'est un stratagème dirigé contre moi. » Dans la conception de l'appareil psychique telle qu'elle s'articule à la deuxième topique, Freud délimitera le champ de la psychose en un conflit entre le moi et le monde extérieur. La perte de la réalité qui est le corollaire des conflits entre l'instance moïque et le monde extérieur, serait donnée au départ de la psychose; en écho, un substitut de réalité serait venu à la place de quelque chose de forclos, témoignant de cette déperdition du sujet qui fait, autour du réel, son spectre fantomatique, son ombre, ses restes en souffrance, débris, morceaux épars comme témoignages d'un désastre identitaire que le délire tentera de reconstruire dans l'après-coup. Objets sans vraie spécificité, substituables mais auxquels Magali s'agrippait de manière fugace, comme autant de débris d'identité qui l'environnaient et qu'elle tentait de faire siens, comme elle pouvait, faute de mieux.

Le processus psychotique concerne la question du passage ou plutôt la question de la traversée d'une expérience-limite énoncée comme suit: "Quand je suis tombée malade, je partais dans la ville, j'errais. Ma maladie a entraîné mon désœuvrement et ma déperdition. » L'errance à travers ce territoire inconnu — à la fois subjectif et spatial — désertique, austère, sans limite, amènera Magali, durant plusieurs semaines, à ne découvrir qu'un désert vide de toute rencontre humaine, alors qu'elle erre dans les rues de la ville nuit et jour.

De cette désolation comme temps d'érosion identitaire et expérience d'un désarrimage ontologique en son chaos morcelé, nous pouvons émettre l'hypothèse que l'épreuve de honte viendrait brutalement comme expression de ce malaise. Honte qui procède elle-même d'une atteinte à la subjectivité et qui, dans son paradoxe même, permet son maintien. Déperdition du sujet et errance comme «hors-lieu» identitaire «diffracté» et éclaté, se soldant par la rencontre avec soimême comme un autre, suivant un schéma narcissique mortifère: «Je ne me reconnais plus... j'ai perdu mon moi...» disait Magali. Face à cette surenchère dans l'exil interne et l'aliénation, la honte comme expérience subjective singulière viendrait d'abord «[...] comme expérience fondamentale de survie, c'est-à-dire une revendication vitale de dignité» (Lévy, 2000, 119). De la solitude à laquelle le sujet se trouve ramené, la honte pourrait à la fois en désigner le symptôme et donc l'appel à l'autre que celui-ci vient signifier.

Ainsi, cette impasse ontologique dont témoignerait «[...] l'urgence éthique de la honte» (Lévy, 2000, 123) nous rappelle que la psychose accomplit son œuvre 
de déterritorialisation: l'être, le plus intime, devient étranger au sujet, de même que le reflet dans le miroir (Magali disait ne plus s'y regarder depuis la maladie) et offre en pâture au regard d'autrui la singularité irréductible d'un sujet exhibé au public dans son intimité et dans sa passivité. Et c'est de cela dont Magali avait honte: «La honte de la folie — écrit S. Tisseron — n'est justement pas la folie, mais l'ultime rempart contre l'envahissement de la personnalité par sa partie folle» (Tisseron, 2006, 30). De telle sorte que l'épreuve de honte fonctionnerait comme expérience éthique, comme un voile de pudeur jeté sur la nudité impudique de l'état de dépersonnalisation, voile censé dissimuler l'intimité de l'être devenu obscène. Mais plus. La honte surviendrait dans cette césure qui clive le sujet, qui provoque une rupture de la pensée et son incapacité structurelle à dire une telle rupture. Dans cette perspective, ne peut-on pas émettre l'hypothèse de la honte comme appel irrépressible à obtenir réponse à cette question du lien à l'autre semblable, la honte comme appel ontologique ? Pour répondre à cette supposition, c'est avec les travaux de Lacan que je propose d'esquisser à présent une compréhension de la honte comme inscription intrinsèque dans la condition humaine ellemême.

Face à l'écrasement de la vie psychique inhérent au processus psychotique - véritable chute sans fin qui leste le sujet entre survie et déshérence s'inscrirait la honte comme fondamentalement ontologique. Formulation capitale puisqu'elle implique de considérer la honte comme épreuve concernant ontologiquement l'être du sujet.

C'est bien là le sens que Lacan donne à $l^{\prime}(h)$ ontologie (Lacan, 1991, 209) qui désigne, dans sa dimension intrinsèque, le réel qui est cause du sujet. C'est ce que découvre la rencontre traumatique dans sa confrontation au réel de la mort, de l'horreur, de l'indicible, de l'insoutenable, au-delà de toute possibilité de symbolisation. Marquée de sa rencontre avec le réel, la honte va imprimer son sceau. C'est là qu'elle s'éprouve, dans son lien à l'originaire et dans le rapport scopique à l'autre semblable: le réel saisi dans le regard d'un autre. En même temps, cette honte ontologique permet au sujet de s'éprouver comme tel, car ce regard qui s'adresse à lui et dont il est le point de mire, manifeste en premier lieu la présence de l'Autre. Pour Lacan, la prise en compte et l'existence de ce regard Autre va modifier le positionnement, le statut du sujet — regardé — qui dès lors devient objet du regard de l'Autre: "Cette fenêtre, s'il fait un peu obscur, et si j'ai des raisons de penser qu'il y a quelqu'un derrière, est d'ores et déjà un regard» (Lacan, 1953-1954, 240). Cette formulation trouve une résonance ontologique et signale la structure phénoménologique de la honte. En d'autres termes, c'est dans le rapport du sujet à l'Autre, dans ce regard Autre qu'il va se voir et se sentir exister ; l'Autre donne consistance au sujet dans sa course désirante : «Car le désir dans sa racine et son essence, c'est le désir de l'Autre [...]» (Lacan, 1960-1961, 212). Lorsque le processus psychotique est venu figer le sujet dans sa sphère psychique et obturer son rapport à la réalité, une limite est irrémédiablement franchie: la honte serait la reconnaissance de ce franchissement, elle témoignerait 
de la lutte à mort symbolique qui est celle de l'ancrage psychotique et de la rencontre avec le réel - la mort proche - exprimée en ces termes par Magali : «J'ai eu un repli de conscience, comme une négation de ma conscience par rapport à la réalité ». Autrement dit, la honte serait liée à un double exil, de cette frontière entre le temps de l'enfance et celui de l'adolescence, entre le temps précédant l'entrée dans la psychose et celui du début de la maladie que Magali évoquait de la manière suivante: «J'ai l'impression d'avoir quitté un passé que je ne retrouverai jamais. J'ai du mal à conserver ces traces... elles ne m'appartiennent plus, c'est comme s'il y avait eu un pillage intime». Confrontée à cette épreuve qui la rend étrange et étrangère à elle-même, la honte ontologique comme épreuve de l'être lui permettrait de s'éprouver comme sujet alors qu'elle se sent de plus en plus souvent dans une errance psychique, à mi-chemin, disait-elle, entre le monde «d'avant que je ne retrouverai jamais » et cet univers étrange, face obscure de la lune, fragments parcellaires psychiques dans lesquels, affirmait-elle, «je ne vis plus comme les autres. Je me sens en dessous des autres tout en y étant, c'est une réalité triste ».

Moment fatidique et radical qui vient scinder le continuum de son histoire, entre «l'avant» et «l'après » de la décompensation psychotique, c'est là aussi que se situe l'émergence dans le réel d'une certaine vérité du rapport à l'autre (Assoun, $1999,54)$ que dès lors le sujet ne pourra plus se masquer: "Je suis différent des autres », répétait Magali durant les entretiens. Il s'agit bien ici de penser l'entrée dans la psychose comme épreuve désastreuse, métamorphose irréversible, impasse d'un sujet écartelé entre un attachement au passé, opaque, sédimenté, et un présent trop douloureux, difficile à accepter, soit entre un déjà plus et un pas encore. En outre, le processus de désymbolisation psychotique entraîne l'impossibilité de ressentir ce qu'on sent et de ne plus disposer alors des mots qui portent pour soimême résonance : «Avec la maladie, le mental en a pris un sacré coup ». Cet insupportable de la souffrance inhérente à l'irréversibilité de cette épreuve, annoncée dans une litanie plaintive itérative au cours des entretiens et qui se manifestait avec acuité dans l'espace transférentiel, mais aussi sur l'incapacité généralisée, sur le handicap mental pressenti, parce qu'ils étaient parlés, introduisaient à la pensée, à la recherche du sens et des causes, et indiquaient chez la patiente, une certaine vigilance de l'être. En ce sens, lorsque l'être humain est confronté à une forme d'indigence contingente à son entrée dans la psychose — «Ma maladie a entraîné ma déperdition », confiait Magali — la honte ontologique concernerait l'essence même de l'Homme et ce qui fonde son appartenance à l'espèce humaine ; en effet, au sens lacanien, la honte est ontologique puisqu'elle est articulée à l'homme. Autrement dit, il est question ici de la «honte de vivre» qui fait écho à la «honte de l'être» au sens de Heidegger pour qui la honte va au-delà d'un sentiment : elle constitue la tonalité émotive qui traverse et détermine tout son être, là où tout signifiant, où le symbolique lui-même s'avère impuissant pour le voiler, être qui se révèle ainsi dans sa face réelle. En ce sens, la honte est une sorte de sentiment ontologique, dont le lieu propre est la rencontre entre l'homme et l'être: «L'être 
lui-même — nous dit Lacan paraphrasant Heidegger — porte avec soi la honte, la honte d'être. » (Lacan, 1991, 211) Il existe donc, pour Heidegger, une honte d'être, qui en fait un affect ontologique dans le sens où la répugnance de celui qui l'éprouve se reconnaît dans l'objet de la répulsion et redoute par là-même d'être reconnu et confirmé par lui.

À la lumière de ces repères théoriques, la honte serait donc à situer au lieu même de la rencontre de l'homme avec son être, et gagne là sa portée structurale, pour ne pas dire universelle : «La honte — conclut le philosophe — est en fin de compte une existence qui se cherche des excuses» (Heidegger, 86, 40). Au sens lacanien, un sujet affecté de la honte de vivre, serait un sujet brutalement dépouillé de ses identifications moïques, surpris par l'Autre dans sa nudité d'être parlant, au point où se pose pour lui la question de l'être, «[...] celle du «pourquoi soi ? » par où le sujet projette dans l'énigme son sexe et son existence» (Lacan, 1957, 450); ou encore en tant que question articulée: « «Que suis-je là ?» concernant [...] sa contingence dans l'être » (Lacan, 1955, 459). À l'appui de ces considérations, la honte d'exister serait à appréhender comme modalité par laquelle «[...] la question de son existence baigne le sujet, le supporte [...] voire «le déchire de toutes parts » (Lacan, 1955, 459). En écho, par la honte comme «signe d'être» (Lacan, 1957-1958, 256) et mode d'appel au regard de l'autre, le sujet témoignerait donc qu'il demeure sujet dans le sens où la honte lui permettrait de s'éprouver comme tel : d'une part elle provoque une blessure d'idéal, fait choir le sujet de ces illusions d'omnipotence narcissique et procède d'une chute dans l'être ; d'autre part elle fait figure de «protestation narcissique» (Assoun, 1999, 109) dans la mesure où «cette peur de «perdre la face» confirme qu'il y a, qu'il reste... une face à perdre» (Assoun, 1999, 109). Point de honte sans sujet.

\section{Pour conclure}

Une remarque de Freud dans son ouvrage Abrégé de psychanalyse va me permettre de conclure mon propos : "Nous disons que dans toute psychose existe un morcellement du moi, et si nous tenons tant à ce postulat, c'est qu'il se trouve confirmé dans d'autres états plus proches des névroses et finalement de ces dernières aussi » (Freud, 1940). J'ai souhaité insister ici, à travers une évocation clinique, sur cette co-occurrence entre honte, psychose et lien social. Il s'agissait, dans cette perspective, de prendre en compte l'ensemble des notions dynamiques en jeu qui, dans leur polysémie, révèlent l'un des paradoxes majeurs de la honte, à savoir qu'elle menace le sujet de disparition, tout en le maintenant sujet. De la solitude à laquelle le sujet - en proie à une désorganisation psychotique - se trouve brutalement confronté, la honte en serait à la fois la cause et en tant que sentiment social, lui permettrait de continuer à s'éprouver comme tel. C'est précisément en ce point que la honte constituerait une expérience subjective contradictoire dans le sens où elle suspend en quelque sorte le sujet entre exclusion et intégration, entre épreuve de déréliction et lien social, entre incertitude narcissique et confirmation existentielle. 
En ce sens, les enjeux de la dynamique intra et intersubjective s'articulent à une dimension destructrice de la honte — elle entame la subjectivité — et l'éprouvé contradictoire du malaise comme mode d'appel au regard de l'autre - maintien de l'identité subjective - dans son caractère fondamentalement paradoxal. En replaçant le sentiment de honte dans l'histoire même du développement psychique, en le délimitant à une de ses phases, en le désignant comme reliquat archaïque se signalant par son discours dans la psychose, l'interprétation théorique et clinique vise à postuler l'expression de honte comme le moyen pour être reconnu comme sujet et objet différencié. Pourtant cette analyse nous laisse sur une énigme dans le sens où une sorte de paradoxe paraît exister entre la honte comme trace dans le temps précédant l'entrée dans la psychose, et la honte comme ne faisant pas trace dans l'expérience psychotique. Je terminerai en citant Magali, lorsqu'elle dit, au terme des entretiens débutés un an plus tôt:

«Cette lucidité sur ma honte est bénéfique

Et à la fois douloureuse... mais à choisir, Je préfère la lucidité que l'ignorance».

delphine scotto di vettimo université de nice sophia-antipolis pôle universitaire saint-jean d'angély 24, avenue des diables bleus 06357 nice cedex 4 dscotto@unice.fr

\section{Références bibliographiques}

Assoun, P.-L., 1999, Le Préjudice et l'Idéal. Pour une clinique sociale du trauma, Paris, Anthropos.

Aulagnier, P., 1984, Telle une zone sinistrée, Adolescence, 2, 1, 9-21.

Freud, S., 1900, L'interprétation des rêves, Paris, P.U.F., 1967.

Freud, S., 1940, Abrégé de psychanalyse, Paris, P.U.F., 1985.

Jacobi, B., 2000, De la honte à la plainte, In Victime-Agresseur Tome 1 - Le traumatisme sexuel et ses devenirs, Lecques, Les Éditions du Champ social, 147-155.

Lacan, J., 1953-1954, Le Séminaire, livre I, Les écrits techniques de FREUD, Paris, Éditions du Seuil, 1975.

Lacan, J., 1960, Subversion du sujet et dialectique du désir dans l'inconscient freudien, Écrits, Paris, Éditions du Seuil, 1966, 793-827.

Lacan, J., 1960-1961, Le Séminaire, livre VIII, Le transfert, Paris, Éditions du Seuil, 1991.

Lacan, J., 1991, Le Séminaire, livre XVII, L'envers de la psychanalyse, Paris, Éditions du Seuil.

Lévy, G., 2000, Au-delà du Malaise, Psychanalyse et barbaries, Toulouse, Érès.

Miollan, C., 1998, Inceste, une écoute post-traumatique, Cliniques méditerranéennes, n ${ }^{\text {os }}$ 55/56, 163-172.

Danger! Le photocopillage détruit les revues. 
Entre honte et psychose : réflexions sur un paradoxe social

Scotto Di Vettimo, D., 2001, Thèse pour le Doctorat en Sciences Humaines. Métapsychologie et clinique de la honte : son statut, ses manifestations, son traitement psychothérapique. Université de Nice Sophia-Antipolis.

Scotto Di Vettimo, D., Jacobi, B., 2003, Du tourment de la honte à la préoccupation narcissique, Psychologie clinique, Paris, L'Harmattan, 16, 111-124.

Tisseron, S., 1992, La Honte. Psychanalyse d'un lien social, Paris, Dunod.

Tisseron, S., 2006, De la honte qui tue à la honte qui sauve, Le Coq-Héron, Secret, honte et violences, La honte à l'épreuve de la psychanalyse, $\mathrm{n}^{\circ} 184,18-31$. 